(62\% female) with CKD (mostly stage 3) and no history of coronary disease. The capacity of the Framingham equation to discriminate between those who would experience a cardiac event and those who would not was poor, with the model correctly identifying only $60 \%$ and $62 \%$ of the men who would experience an event within 5 or 10 years, respectively. Discrimination in women was better, ranging from $77 \%$ for 5 -year events to $73 \%$ for 10-year events.

Discrimination was improved by re-weighting the Framingham equation covariates, indicating that traditional risk factors are relevant in individuals with CKD, but that the relative importance of each factor differs between CKD and non-CKD populations. Recalibration to account for systematically higher event rates in the study population than in the original Framingham cohort improved predictive accuracy in women, but not in men, possibly because of a particularly high incidence of the competing outcome of death in men with CKD.

The researchers conclude that although the Framingham equation can be adjusted to better predict incident coronary disease in individuals with CKD, new CKD-specific equations are required.

Original article Weiner DE et al. (2007) The Framingham predictive instrument in chronic kidney disease. J Am Coll Cardiol 50: 217-224

\section{The treatment of severe lupus nephritis with mycophenolate mofetil-a meta-analysis}

The immunosuppressant mycophenolate mofetil (MMF) is associated with fewer adverse effects than other therapies for lupus nephritis, but results of randomized controlled trials (RCTs) in severe forms of the disease have been conflicting. Following a systematic literature review and meta-analysis of published RCTs, Zhu and colleagues have concluded that MMF is superior to pulsed intravenous cyclophosphamide as induction therapy for severe lupus nephritis, and a feasible alternative to azathioprine for maintenance therapy.

MEDLINE, EMBASE and Cochrane Collaboration Database searches identified five RCTs (307 patients) for inclusion. Four trials yielded data on induction therapy, comparing
MMF plus a steroid with cyclophosphamide plus a steroid, and two compared maintenance therapy of MMF plus a steroid with azathioprine plus a steroid. Overall, MMF significantly reduced the risk of infection compared with cyclophosphamide (relative risk 0.65; $P<0.001)$. Patients administered MMF had a markedly increased complete remission rate (relative risk 3.10; $P=0.006$ ), and a reduced risk of leukopenia (relative risk 0.66; $P=0.04$ ) compared with those given cyclophosphamide. The risks of developing end-stage renal disease, death, or experiencing other adverse effects were not notably different between those receiving MMF and those receiving cyclophosphamide. When compared with azathioprine for maintenance therapy, MMF did not significantly affect prognosis or risk of amenorrhea or herpes zoster. Gastrointestinal events were more frequent with MMF than with the other two agents, but the differences were not significant.

Original article Zhu B et al. (2007) Mycophenolate mofetil in induction and maintenance therapy of severe lupus nephritis: a meta-analysis of randomized controlled trials. Nephrol Dial Transplant 22: 1933-1942

\section{Enzyme replacement therapy in patients with Fabry disease and end-stage renal disease}

Fabry disease is an X-linked disorder in which defective $\alpha$-galactosidase A results in globotriaosylceramide accumulation in the kidney and other organs. Most affected males progress to end-stage renal disease (ESRD) during midlife; dialysis and transplantation are effective treatments for the renal, but not the systemic, problems. Enzyme replacement using recombinant human a-galactosidase A (e.g. agalsidase alfa) is now an option in patients with Fabry disease, but data on its use in those with ESRD are limited.

A recent study investigated the safety and first-dose pharmacokinetics of agalsidase alfa in 21 patients (19 male) with ESRD who were on dialysis $(n=8)$ or had undergone kidney transplantation $(n=13)$. Patients received standard-dose agalsidase alfa (one $0.2 \mathrm{mg} / \mathrm{kg}$ infusion over 40 minutes every second week) for a median of 42 weeks.

Plasma globotriaosylceramide levels had decreased significantly after approximately 